\title{
Tickende Zeitbombe „Bauchaortenaneurysma“
}

\section{Ultraschall-Screening für Männer über 65 Jahre ab sofort Kassenleistung}

Seit Beginn des Jahres haben gesetzlich krankenversicherte Männer ab 65 Jahren Anspruch auf ein einmaliges Ultraschallscreening zur Früherkennung von Bauchaortenaneurysmen. Die Deutsche Gesellschaft für Ultraschall in der Medizin e. V. (DEGUM) begrüßt diese Entscheidung, da insbesondere Männer in diesem Alter ein erhöhtes Risiko für diese gefährliche und häufig unerkannte Erkrankung aufweisen. Die Fachgesellschaft fordert jedoch, auch Frauen sowie Patienten ab 55 Jahren mit einem besonderen Risikoprofil, wie etwa Diabetes oder Rauchen, einzubeziehen.

An einem Bauchaortenaneurysma erkranken etwa 4 bis 8 Prozent aller Männer über 65 Jahren. Dabei ist die Früherkennung ausschlaggebend dafür, ob der Patient diesen Vorfall überlebt. Denn bei einer Aussackung des größten Gefäßes im Bauchraum bleibt der Patient zunächst meist beschwerdefrei. „Wenn die Bauchaorta infolge der Aussackung reißt, kann der Betroffene innerhalb kürzester Zeit verbluten“, warnt Professor Dr. med. Thomas Fischer, Leiter des Interdisziplinären Ultraschall-Zentrums des Instituts für Radiologie am Campus Charité BerlinMitte. Untersuchungen zeigen, dass nur etwa 10 Prozent der Patienten mit einem Riss in der Bauchaorta lebend im Krankenhaus ankommen. „Ein anderes Risiko besteht darin, dass infolge der Gefäßvergrößerung ein Blutgerinnsel zu Gefäßverschlüssen in den Beinen führen kann.“
Aufgrund dieser Gefahrenlage für viele Patienten begrüßt die DEGUM die Entscheidung der Kassenärztlichen Bundesvereinigung (KBV) und der Gesetzlichen Krankenversicherung (GKV), Männern über 65 eine Früherkennungsuntersuchung per Ultraschall zu gewähren. Im Zuge dessen kann ein Bauchaortenaneurysma schnell erkannt und operativ behandelt werden, um gefährliche Folgeschäden zu vermeiden. Dies kann mittels des Einsatzes einer Gefäßprothese oder in Einzelfällen mit einer Stent-Prothese erfolgen.

Das Screening soll im Rahmen der Gesundheitsuntersuchung „Check-up 35“ einmalig erfolgen. Diese dürfen Hausärzte, Urologen, Internisten mit und ohne Schwerpunkt, Chirurgen und Radiologen durchführen. Hierfür sollten Untersucher eine nachweisbare Qualifikation haben, beispielsweise ein DEGUMZertifikat der Stufe 1, empfiehlt Fischer, der auch stellvertretender Leiter der DEGUMSektion Radiologie ist. Denn es gehe nicht nur darum, den Durchmesser der Bauchaorta zu bestimmen, sondern auch einen Einriss oder ein Aneurysma des Gefäßes frühzeitig zu erkennen. Ärzte, die keine Genehmigung für eine Bauchaorten-Sonografie haben, können dennoch das Aufklärungsgespräch führen und dies separat mit den Krankenkassen abrechnen.

Auch wenn Männer häufiger von einem Bauchaortenaneurysma betroffen sind als Frauen, sollten letztere nach Ansicht der DEGUM auch von der Vorsorgeuntersu- chung profitieren können - insbesondere Patientinnen mit erhöhtem Risiko wie Artheriosklerose. „Es wäre insgesamt ratsam, Patienten mit einem besonderen Risikoprofil - beispielsweise bei Diabetes, Bluthochdruck, einer Fettstoffwechselerkrankung sowie starke Raucher ab dem 55. Lebensjahr - in das Vorsorgescreening einzuschließen“, sagt Professor Fischer.

\begin{abstract}
Information: Welche
Beschwerden verursacht ein Bauchaortenaneurysma?

Das Gefährlichste am Bauchaortenaneurysma ist, dass es vom Patienten meistens nicht bemerkt wird. Durch Größenzunahme der Bauchaorta können vor allem Rückenschmerzen auftreten. Häufig führt dies zu Beschwerden im Bereich der Wirbelsäule oder der Nieren. In den Fällen, in denen ein Bauchaortenaneurysma platzt, wird vom Patienten ein unerträglicher Bauchschmerz mit Ausstrahlung in den Rücken, Übelkeit und Brechreiz bemerkt. Durch die innere Blutung kommt es zu einer lebensbedrohlichen Situation.
\end{abstract}

\section{Quellen:}

http://www.kbv.de/html/1150_32008.php https://www.krankenkassenzentrale.de/ magazin/ultraschalluntersuchung-zur-frueherkennung-von-bauchaortenaneurysmen-kuenftig-gkv-leistung-4173\# 